Bangladesh J. Bot. 49(2): 287-296, 2020 (June)

\title{
EVOLUTIONARY AND PHYLOGENIC RELATIONSHIPS OF WILD AND CROP SPECIES OF IRANIAN SAFFRON BY DNA BARCODING
}

\author{
Farshid Golshani*, Brat Ali Fakheri, Mahmood Solouki, \\ Nafiseh Mahdinezhad and MaJid Reza Kiani Feriz ${ }^{1}$ \\ Department of Plant Breeding and Biotechnology, Faculty of Agriculture, \\ University of Zabol, Iran
}

Keywords: Phylogenic relationship, Wild and crop species, Crocus, DNA barcoding

\begin{abstract}
DNA barcoding method was applied to identify and study the phylogenic relationships existing between 13 species of saffron collected in Iran including 4 crop and 9 wild species. PCR amplifications were performed using primers designed on the nucleotide sequence of three plastid barcode genes, comprising two protein encoding genes (rbcL and matK) and an intragenic spacer (trnH-psbA), and a nuclear region (ITS). A total of 52 sequences were obtained and registered in NCBI database. In particular, 21 of these sequences were not present in the scientific library. Nucleotide polymoprhic sites were counted for each barcode gene ( $\mathrm{rbcL}, \mathrm{n}=16$; matK, $\mathrm{n}=15$; trnH-psbA, $\mathrm{n}=46$; ITS, $\mathrm{n}=71$ ). Each sample could be distinguished from the others in the phylogenic trees developed based on the data obtained by single barcode gene. In addition, a phylogenic tree based only on plastid information (trnH-psbA + rbcL + matK) and another created on the data resulting from both nuclear and plastid genomes ( $\operatorname{trnH}-\mathrm{psbA}+\mathrm{rbcL}+$ matK + ITS) was also generated. In general, ITS sequence, indicating high resolution at the genus and species level, appeared as the best barcode sequence of the present study. Phylogenic analysis demonstrated the genetic relationship between crop saffron and wild Crocus species. According to the results of this study, among 13 available sample, the wild species are Crocus cancellatus L. and Crocus sp. Eslamabad were hypothesized as the closest species to the Iranian saffron. The present investigation also indicated that the different ecotypes of $C$. sativus L. may have evolved through independent events probably due to geographic and environmental pressures.
\end{abstract}

\section{Introduction}

Crocus L. is a monocot belonging to Iridaceae family (Mathew 1982), Crocus series Crocus, which is distributed from Italy in the West to the Caucasus in the East with the center of diversity on the Balkan Peninsula and Asia Minor (Larsen et al. 2015). Only one species of this genus is source of saffron (C. sativus). It is triploid and its stigma, picked up manually, is desiccated for the production of saffron (the world's most expensive spice) (Zubor et al. 2004). Other species of saffron have attracted attention as garden plants and collection species (Petersen et al. 2008). In molecular biology, DNA barcode technique is a highly accurate scientific tool for taxonomic identification at species level, which is based on the high rate of mutations occurring in target sequences during the evolution (Gismondi et al. 2012). This method, first proposed by Hebert et al. (2003), shows a simple relationship between all stages of life at levels beyond species. The Consortium for the Barcode of Life is trying to develop this method (Casiraghi et al. 2010). The standard nucleotide sequence analysis (matK, maturase K; rbcL, RuBisCO large subunit; trnHpsbA, intragenic spacer between tRNAHisGUG gene and thylakoid membrane protein of photosystem II of Mr 3200 gene; ITS, internal transcription spacer of nuclear ribosomal DNA) has been successfully used to identify and classify several plant species (Gismondi et al. 2012, 2013).

Petersen et al. (2008), for the first time, performed phylogenetic analysis on Crocus genus using three nucleotide sequences encoding proteins (ndhF, accD, and rpoCl) and two other non-

*Author for correspondence: <farshidgolshani@gmail.com>. ${ }^{1}$ Department, Khorasan Razavi Agricultural and Natural Resources Research and Education Center, AREEO, Mashhad, Iran. 
encoding regions (trnH-psbA and rpl36-rps8) of the plastid genome. Harpke et al. (2013) analyzed phylogenic relationships and evolution samples of Crocus, via trnL-F, ITS, and pCOSAt103 barcodes, demonstrating ITS/trnL-F data resulted in a monophyletic genus Crocus, and cloning of pCOSAt103 resulted in the detection of homoeologous copies in about one third of the taxa of section Nudiscapus, indicating an allotetraploid origin of this section. Gismondi et al. (2013) studied Italian and Spanish saffron species, through ITS, rbcL matK, and trnH-psbA barcodes demonstrating independent event of generation of Crocus sativus species in Spain and in Italy. The purpose of this study is to study the best barcode for identifying Crocus species and evolution as well as phylogenic relationships between some wild and crop species of Iranian saffron by DNA barcoding approach.

\section{Materials and Methods}

The plant material was collected from 9 wild Crocus species including $C$. cancellatus, $C$. michelsonii, C. speciosus deylaman, C. speciosus roudbar, C. caspius, C. almehensis, C. sp. Eslamabad-e Gharb, Crocus sp. harsin, and $C$. sp. sonqor, as well as four crop species including $C$. sativus fars, $C$. sativus khorasan razavi, $C$. sativus nehbandan, and $C$. sativus zabol, in different regions of Iran. Detailed information is presented in Table 1. For each specimen, 10 samples were considered and analyzed. These plant species cover most wild and crop species in Iran. The experiment was conducted at the Center of Agricultural Biotechnology, University of Zabol, Iran.

Table 1. Scientific name, specification and place of collection of saffron species used in this study.

\begin{tabular}{lllllll}
\hline Species & $\begin{array}{l}\text { Flowering } \\
\text { time }\end{array}$ & County & Altitude & Latitude & Longitude & $\begin{array}{l}\text { Number of } \\
\text { samples }\end{array}$ \\
\hline C. cancellatus & Autumn & Kermanshah & 2055 & 34.39 & 46.56 & 10 \\
C. caspius & $"$ & Rasht & 161 & 37.44 & 49.96 & 10 \\
C. speciosus & $"$ & Roudbar & 1340 & 36.50 & 49.43 & 10 \\
C. speciosus & $"$ & Deylaman & 1573 & 36.52 & 49.05 & 10 \\
C. sp. & $"$ & Harsin & 1542 & 34.11 & 47.27 & 10 \\
C. sp. & $"$ & Sonqor & 1805 & 34.39 & 47.41 & 10 \\
C. sp. & $"$ & Eslamabade Gharb & 1350 & 34.07 & 43.36 & 10 \\
C. michelsonii & Spring & Bojnurd & 1376 & 37.27 & 57.18 & 10 \\
C. almehensis & $"$ & Hamedan & 1850 & 34.75 & 48.53 & 10 \\
C. sativus & Autumn & Torbat-e Heydarieh & 1354 & 35.20 & 59.22 & 10 \\
C. sativus & $"$ & Estahban & 1720 & 29.07 & 54.02 & 10 \\
C. sativus & $"$ & Nehbandan & 1100 & 31.48 & 60.01 & 10 \\
C. sativus & $"$ & Zabol & 475 & 31.04 & 61.53 & 10 \\
\hline
\end{tabular}

DNA was extracted from Crocus sp. leaves by Dellaporta method (Dellaporta et al. 1983). Purified DNA was amplified using PCR method in a reaction $50 \mu \mathrm{l}$ mixture containing $2 \mu \mathrm{l}$ of DNA template, $2 \mu \mathrm{l}$ of each primer (Table 2) with a concentration of 10 picomolar and $25 \mu \mathrm{l}$ of Master Mix 2x (Ampliqon, Germany). For the final volume, deionized distilled water was used. DNA was replicated using BioRad ICycler. The PCR products were separated on agarose gel 1\%, using buffer TAE 1X by adding Fluoro Vue Nucleic Asid Gel Stain $2 \mu$ molar 10000X (Smobio) and visualized under UV light. All PCR reactions were performed at least in triplicate.

All amplicons were sequenced by Macrogene (South Korea). The sequence quality was evaluated using software DNASTAR (Schwei 2015) SeqMan. The sequences were visualized by BioEdit v7.0.5 program. Barcode genes of each sample were compared using Clustal W2-Multiple sequence alignment individually (rbcL, matK, trnH-psbA, and ITS) or in combination (trnH-psbA 
+ rbcL + matK; rbcL + matK + trnH-psbA + ITS). All detectable variable sites were included in the analysis. In order to certify the amplifications, the identity of each amplified sequence was compared to that of those already registered in GenBank (NCBI) using sequence alignment search tool BLAST. The results of this study resulted in the registration of 52 sequences for Crocus species on NCBI site (Table 3). Phylogeny analysis was performed through MEGA5 software (Tamura et al. 2011). In software MEGA5, all necessary parameters were set as reported in Huang et al. (2016). The genetic tree was drawn applying Tamura-Nei model, maximum likelihood method, where 1000 bootstrap validation system and genetic indices were examined using DNA SP. An orchid case (Orchis mascula, ID: JN896032, JN893527, HG800547, AY351379), extrapolated by NCBI database, was also added to this analysis as out-group species.

Table 2. Primer pair names ( $F$ : forward; $R$ : reverse), their sequences, target gene and relative $\mathrm{Tm}(\mathrm{C})$ used in PCRs.

\begin{tabular}{llcc}
\hline Primer pair name & Sequence $^{\text {a }}\left(5^{\prime}-3^{\prime}\right)$ & Amplified gene & Tm (C) \\
\hline rbcL F & ATGTCACCACAAACAGAGACT & rbcL & 57.4 \\
rbcL R & TGTCCATGTACCAGTAGAAGA & & \\
matK F & GTTCTAGCACAAGAAAGTCGA & matK & 56 \\
matK R & CTCAGATTATGATATTATTGA & & \\
trnH-psbA F & CGCGCATGGTGGATTCACAATCC & trnH-psbA & 57.4 \\
trnH-psbA R & GTTATGCATGAACGTAATGCTC & & \\
ITS F & TCCTCCGCTTATTGATATGC & ITS & 57.5 \\
ITS R & CCTTATCATTTAGAGGAAGGAG & & \\
\hline
\end{tabular}

${ }^{\mathrm{a}}$ References: Gismondi et al. (2012, 2013).

Table 3. The results of this study resulted in the registration of 52 sequences of ITS, rbcL, matK and trnH-psbA for Crocus species on NCBI site.

\begin{tabular}{llll}
\hline ITS & rbcL & matK & trnH-psbA \\
\hline KY860629 & MF034878 & MF066223 & KY990029 \\
KY860628 & MF034877 & MF066222 & KY990028 \\
KY860627 & MF002371 & MF066216 & KY990024 \\
KY828970 & MF002366 & MF066211 & KY923248 \\
KY797651 & MF034876 & MF066221 & KY962510 \\
KY797650 & MF034875 & MF066220 & KY990022 \\
KY886374 & MF034874 & MF066219 & KY990025 \\
KY886373 & MF034873 & MF066218 & KY990026 \\
KY886372 & MF002369 & MF066214 & KY996723 \\
KY797649 & MF002372 & MF066217 & KY990023 \\
KY797648 & MF002370 & MF066215 & KY990027 \\
KY860626 & MF002368 & MF066213 & KY923250 \\
KY614361 & KY695236 & KY695238 & KY645945 \\
\hline
\end{tabular}

The concatenated alignment of rDNA regions had a length of 293 bp (Table 4) and was the most variable dataset with 20 parsimony informative sites (PIS), while the concatenated chloroplast data (721 bp) had the fewest PIS (8). Aligning the four concatenated loci resulted in the alignment of 1014 bp length with 28 PIS (Table 4). The results of conserved DNA regions of 
the ITS, chloroplast data, and combined gene in the Crocus family were 0.79, 0.98 and 0.93 , respectively. Conservation threshold region revealed a minimum conservation length of 61,39 , and 53 bases respectively, and sequence conservation of $0.694,0.886$, and 0.836 , respectively (Table 4). These conserved regions included a small part of the sequence of the above gene, suggesting a different differentiation of this position as well as its susceptibility to nucleotide changes and mutations among different varieties, culminating in variability among the variants.

Table 4. Characteristics of the included data partitions.

\begin{tabular}{lcccccccccc}
\hline $\begin{array}{l}\text { Character } \\
\text { partition }\end{array}$ & $\begin{array}{c}\text { Alignment } \\
\text { length }\end{array}$ & $\mathrm{S}$ & $\mathrm{H}$ & $\mathrm{Hd}$ & $\mathrm{Pi}$ & $\mathrm{Eta}$ & $\mathrm{K}$ & $\mathrm{C}$ & $\mathrm{MWL}$ & $\mathrm{CT}$ \\
\hline ITS & 293 & 71 & 9 & 0.945 & 20 & 85 & 17.505 & 0.694 & 61 & 0.79 \\
$\begin{array}{l}\text { trnH-psbA + } \\
\text { rbcL + matK }\end{array}$ & 721 & 77 & 11 & 0.934 & 8 & 83 & 13.437 & 0.886 & 39 & 0.98 \\
Combined & 1014 & 148 & 13 & 0.989 & 28 & 168 & 30.978 & 0.836 & 53 & 0.93 \\
\hline
\end{tabular}

$\mathrm{S}$ : The number of polymorphic positions, H: number of haplotypes, Hd: Haplotype (gene) diversity, Pi: Parsimony informative sites, Eta: total number of mutations, K: number of nucleotide differences between population or species (nucleotide divergence), C: Sequence conservation, MWL: Minimum conservation Length, CT: Conservation threshold.

\section{Results and Discussion}

The success rate of rbcL gene amplifications was calculated as $85 \%$ in Crocus plant samples. Also, $80 \%$ of them were successfully sequenced. The length of each amplicon was verified on agarose gel (Fig. 1). Then, all sequences were verified by BLASTn, with each one of them completely fitting other rbcL sequences of crocus species registered in GenBank. After searching the database, it was found that 9 rbcL sequences, of 13 submitted to NCBI, had not been previously recorded in the database and, therefore, they were first recorded in NCBI database (MF034876, MF034875, MF034874, MF034873, MF002369, MF002372, MF002370, MF002368 and KY695236). In the phylogenetic tree obtained from rbcL barcode data, the species related to saffron genus (C. sativus) were separated from the others (Fig. 2). The number of variable sites (SNPs) was counted as 16 using rbcL sequences in this study.

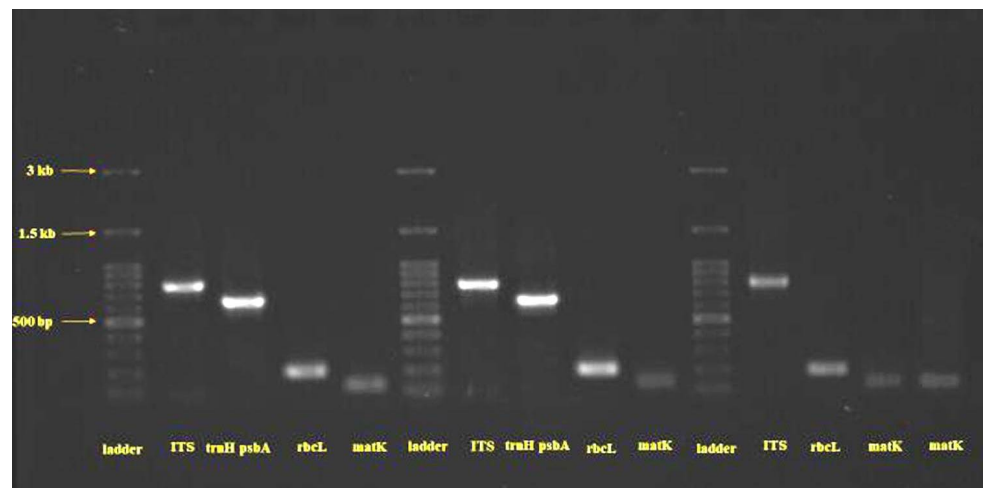

Fig. 1. DNA extracted from a saffron sample, amplified by PCR and visualized, by UV light, after separation on $1 \%$ agarose gel. trnH-psbA, rbcL, matK and ITS barcode genes were shown. Molecular weight markers were also reported (ladder lane). 
The success rate of trnH-psbA gene amplification was calculated to be $92 \%$. Here, $85 \%$ of the amplicons were successfully sequenced. The length of each amplification was verified on agarose gel (Fig. 1). The results obtained by the sequence alignment with Nucleotide-NCBI database by BLASTn confirmed the genetic identity of trnH-psbA sequences of crocus species. Here, 13 sequences of trnH-psbA for these species were deposited in the NCBI site. After searching the databases, it was found that 5 of them had not already been registered in the database (KY990023, KY990027, KY990025, KY990026 and KY996723). According to trnH-psbA sequences, the resulted phylogenic tree revealed a relationship in Crocus genus as reported in Fig. 3. The number of variable sites was counted to be 46 in this case.

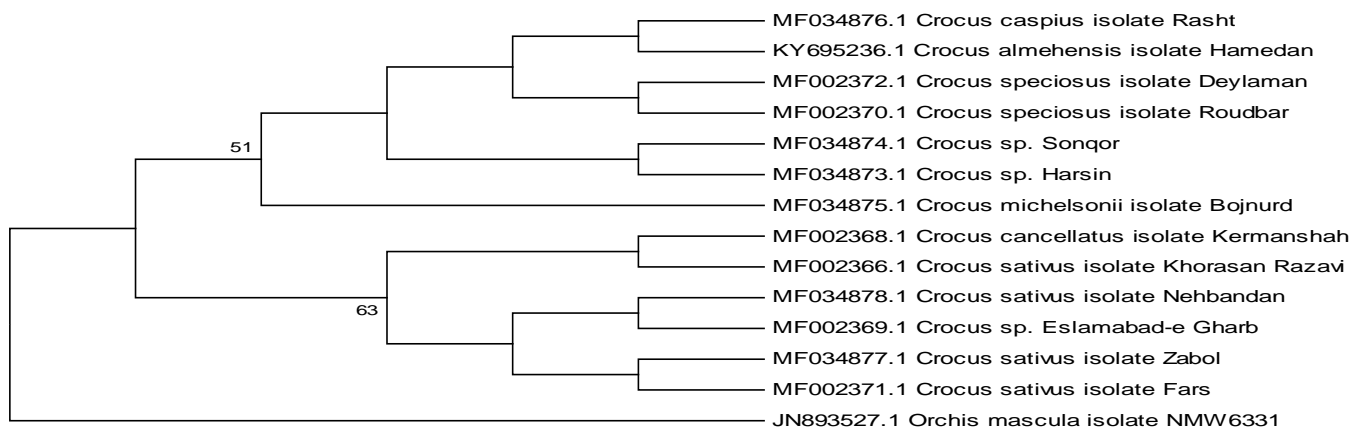

Fig. 2. Phylogenic tree showing the relationship constructed from rbcL sequences of Crocus species.

The matK gene amplification success rate was found to be $80 \%$ in the studied crocus samples, where $73 \%$ of them were effectively sequenced. Each amplification length was authenticated on agarose gel (Fig. 1). In order to verify the identity of the matK sequences resulting from Crocus samples, the obtained sequences were certified by employing BLASTn and through GenBank accessions. The results of the present study led to the registration of 13 matK sequences for these species on NCBI site. Having investigated the database, it was realized that 3 matK sequences observed in the present research had not been previously registered in the database and were accordingly deposited in the NCBI database (MF066214, MF066218 and MF066219). Furthermore, a phylogenic tree was produced according to the matK data (Fig. 4). Using matk sequences, the number of variable sites was counted to be 15 (SNPs).

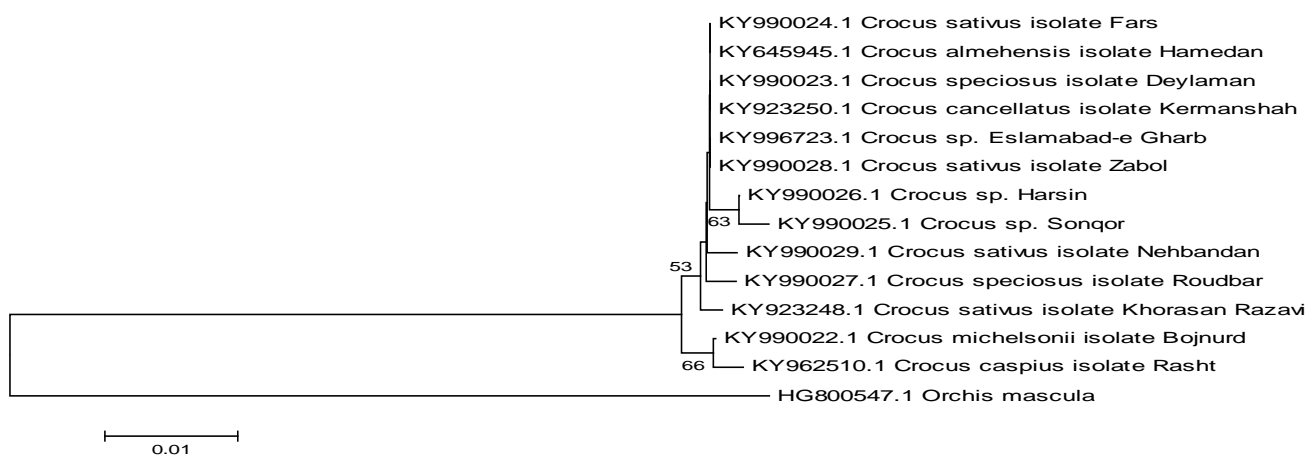

Fig. 3. Phylogenic tree showing the relationship constructed from trnH-psbA sequences of Crocus species. 
The ITS region amplification success rate was measured to be $94 \%$ in the examined Crocus plant samples, where $93 \%$ of them were efficaciously sequenced. The amplicons length was verified on agarose gel (Fig. 1). All the sequences were examined by using BLASTn and through GenBank database and all of them were attributed to other ITS sequences of Crocus species which had already been submitted to NCBI. The results of the present study corresponded to 13 ITS sequences available on NCBI site. After analyzing the database, the researchers observed that 4 ITS sequences found in the present study had not been recorded in the database. Therefore, these sequences were successfully deposited in the database (KY797650, KY886374, KY886373 and KY886372). According to the ITS barcode data, crocus genus species were strongly separated in the phylogenic tree (Fig. 5). It was measured that there were 71 variable sites for the specific ITS sequences obtained in this research (SNPs).

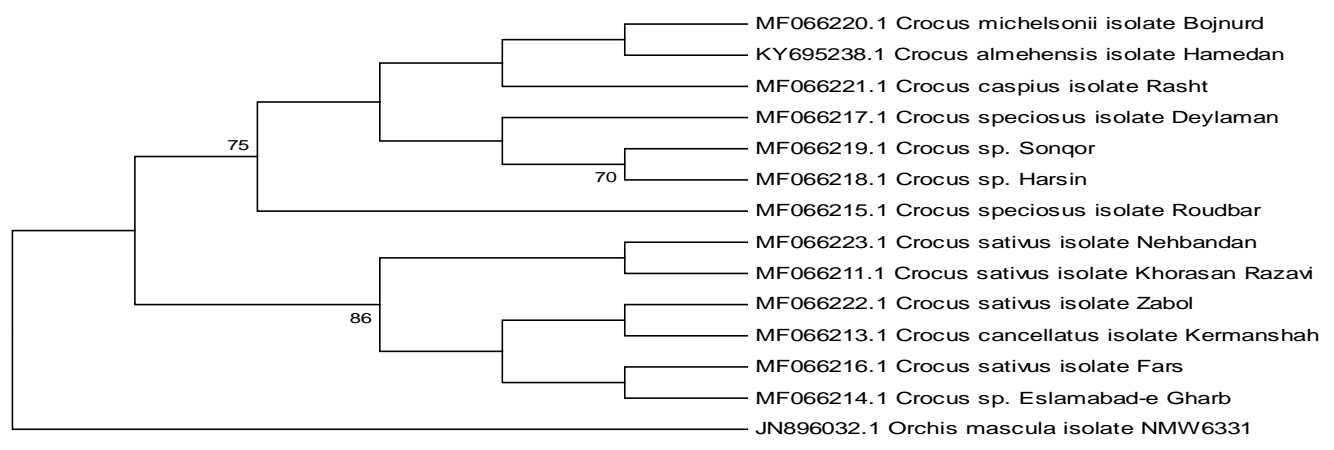

Fig. 4. Phylogenic tree showing the relationship constructed from matK sequences of Crocus species.

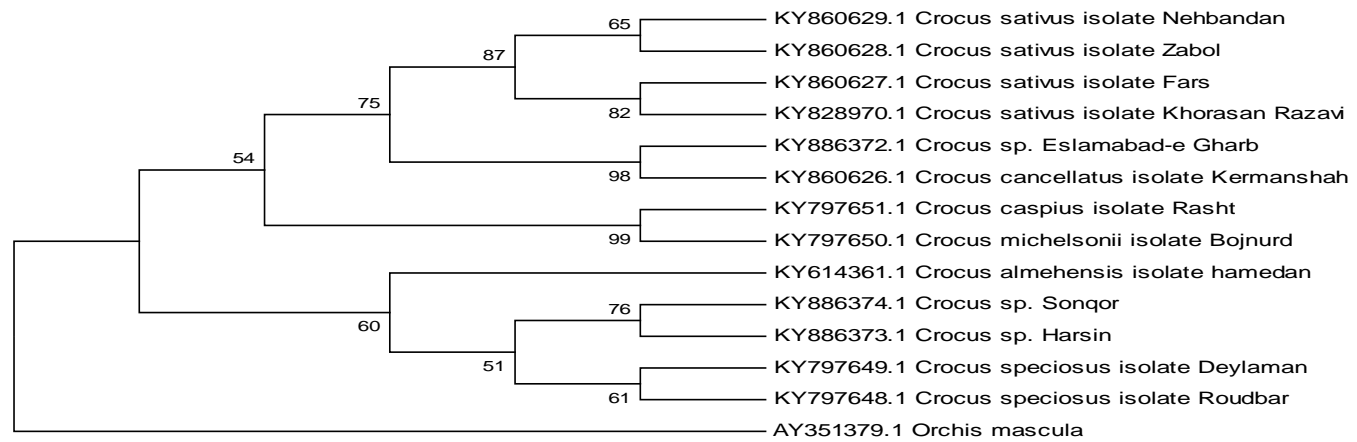

Fig. 5. Phylogenic tree showing the relationship constructed from ITS sequences of Crocus species.

Different cultivars of herbal species and their wild relatives constitute a large part of the valuable plant samples of each country. Today, various hazards such as drought, overgrazing, and over-harvesting have endangered genetic reserves of these plants (Rahimmalek et al. 2009). Genetic identification and registration of various plant cultivars is crucial for adequate conservation and utilization of genetic resources; a monitoring which is hard to be performed by morphological features for most plants in the early stages of development (Asadi et al. 2015). According to the literature, a barcode should show a good resolution power at interspecific levels (Hebert et al. 2003). 
The ITS nuclear barcode used in this study, which has high resolution at both genus and species levels, has been widely used in literature as testified by the elevated number of accessions registered in scientific database under this name. Although it has different lengths among plant organisms, ITS barcode has been adopted as its flanking regions are strongly conserved and allow the use of generic primers. Further, as it is present in multiple copies per cell, it represents a good candidate for plants' barcoding (Kress et al. 2005). Indeed, nuclear genome deals with more changes, which is more informative than plastid genome, and therefore, it is more useful in interspecies studies (Kim and Misra 2007).

RbcL barcode was reported to be informative in identifying Crocus plant samples, showing low evolution rate but high-resolution level during alignment with related genera (Kress et al. 2009). Although rbcL has low resolution at the species level, most studies emphasize that it can strongly contribute to interspecific studies in combination with other plant barcodes (Kress et al. 2005).

Considering the high success rate of amplification and sequencing of trnH-psbA barcode, this specific region was suggested as a strategic tool for plant species classification. The disadvantages of trnH-psbA sequence, even evidenced in this study, are presence of repetitive positions and inappropriate sequence alignment due to its variable length among samples. In the study of Kress et al. (2005), this genome site has been introduced along with ITS as a good candidate for plant barcoding.

Due to the high evolutionary rate and extensive presence among plants, matK gene has been extensively used in phylogenic studies (Rohwer 2000, Kim et al. 2007). At the same time, this sequence has always indicated high amplification and sequencing rate, suggesting it as an optimal target for genetic studies (Wolfe et al. 1987). This phylogenetic reconstruction seemed to enjoy greater discrimination power compared to rbcL and trnH-psbA data previously produced in this work.

In crop species of saffron, which are replicated through basal maternal, the plastids transmitted through the maternal cytoplasm should play an important role. Indeed, using a combination of plastid barcodes, more relevant results should be obtained. In this regard, Peterson et al. (2008), for the first time, performed the sequencing of five plastid genes (trnH-psbA, rp136rps8, ndhF, accD, and rpoC1) on Crocus samples and used all of them to solve specific genetic questions about this genus. Similarly, Hollingsworth et al. (2009) successfully used a combination of plastid genes (rbcL, matK, trnH-psbA, and rpoC1) on angiosperms and gymnosperms plant groups. In particular, in order to provide more accurate information, a phylogenetic tree, including the entire trnH-psbA+rbcL+matK data was created (Fig. 6).

The phylogenic hypothesis that is based solely on the plastid sequences cannot be totally complete, as in cases of hybridization it only reflects the plastid evolution typical of only one parent (Petersen et al. 2008). Rohwer (2000) also states that diversity in the sequence of one or two plastid genes is not sufficient to determine the genetic linkage among species, since in some plant's hybridization and back-crossing also occur (Okuyama et al. 2005).

According to all this evidence, in this study, three plastid sites including two protein encoding genes (rbcL and matK) and one intergeneric spacer (trnH-psbA), as well as a nuclear sequence, ITS, were used to reconstruct phylogenetic events in Crocus genus, using plant materials taken from Iran. These sites were selected because of the relatively high ability to diversify Crocus species (Gismondi et al. 2013). Meanwhile, all these sites have also been proposed as barcoding candidates for plant organisms (Kress et al. 2005; Peterson et al. 2008). Accordingly, we performed our analysis using the three plastid genes and joined them to the ITS sequence (Fig. 7). 
In the present result, a dual separation can be seen. Obviously, Orchis mascula, as expected, positioned itself far away from all other saffron species, being the outgroup. Crocus genus appeared monophilic according to the present data. Accepting this hypothesis, a basic branching divided all samples in two clades, which were poorly supported and poorly sustained (bootstrap values of 52 and $63 \%$ ), respectively.

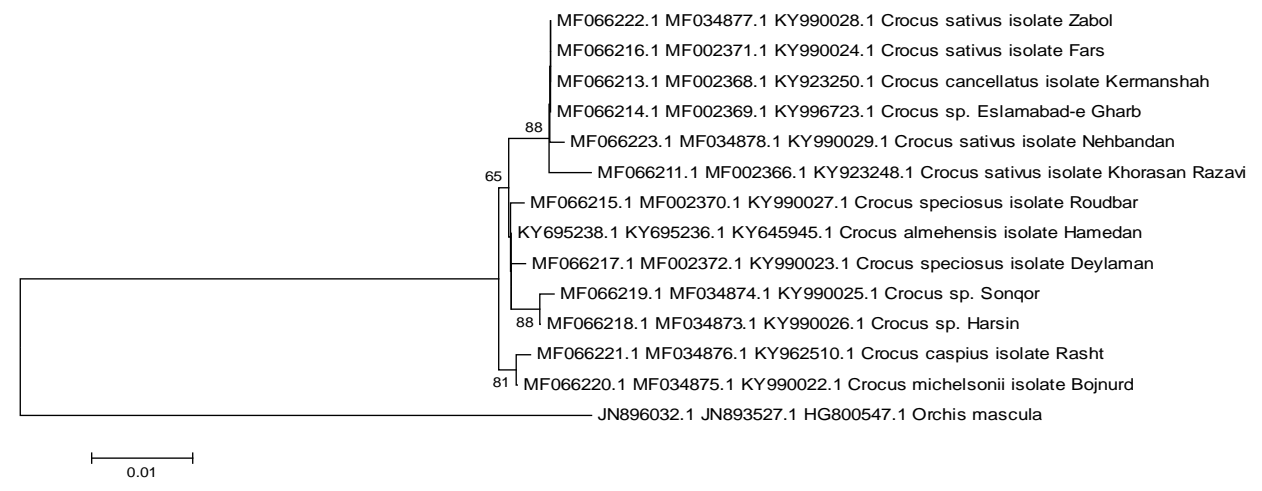

Fig. 6. Phylogenic tree showing the relationship constructed from trnH-psbA+rbcL+matK sequences of Crocus species.

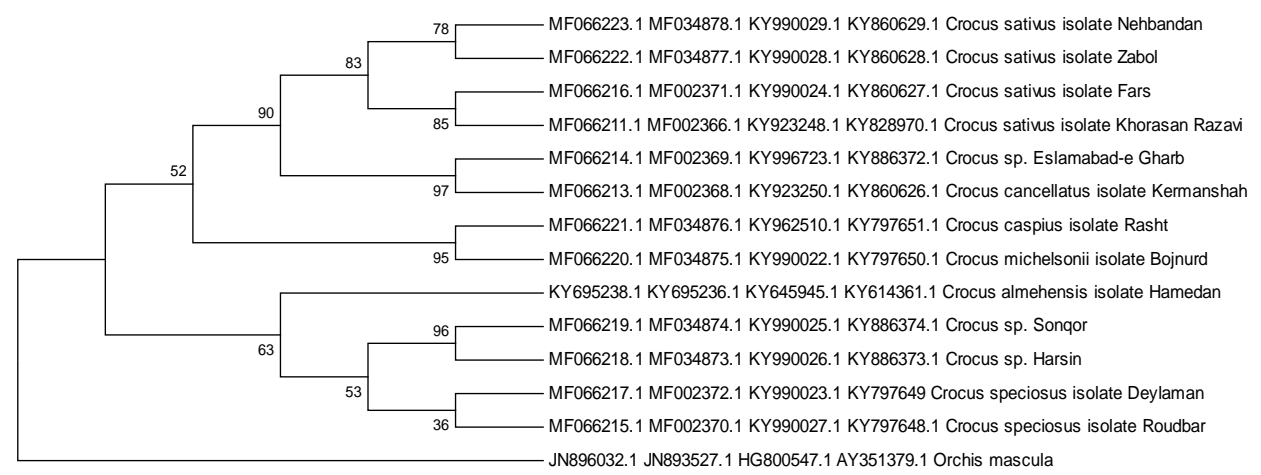

Fig. 7. Phylogenic tree showing the relationship constructed from trnH-psbA+rbcL+matK+ITS sequences of Crocus species.

The first clade had a two-part separation in the base.

The first part was made up of a strongly supported clade (bootstrap value of 90\%) which included all $C$. sativus samples, with the exceptional addition of $C$. cancellatus and another unidentified $C$. species. Specifically, Crocus sativus series formed a single group strongly supported (Bootstrap 83\%) which could be divided in media and strongly sustained clusters (bootstrap values 78 and 85\%, respectively), including C. sativus isolate Nehbandan and C. sativus isolate Zabol (1st cluster), and C. sativus isolate Fars and C. sativus isolate Khorasan Razavi (2nd cluster). Since both these last clades included crop species which were replicated asexually, this important result suggested that some $C$. sativus species might have been generated by different and independent evolutionary events, probably due to different geographic and environmental pressures, as already proposed by Gismondi et al. (2013). Indeed, C. sativus is thought to be a hybrid evolved by breeding between $C$. cartwrightianus and another Crocus species (Zubor et al. 
2004). The series belonging to Nudiscapus and Reticulati subgenus (C. sp. eslamabad-e Gharb and C. cancellatus) were included into this clade with strong validation (bootstrap value of $97 \%$ ).

The second portion of the first clade, presenting species from Orientales and Biflori series $(C$. michelsonii and C. caspius, respectively), was characterized by a strong bootstrap value (95\%). Morphologically, C. caspius is not a typical member of Biflori series. Consequently, its position in the phylogenetic tree was not so surprising. Crocus caspius and Orientales series have the same unusual behavior, with capsules reaching the ground; thus, their close genetic relationship can be easily explained.

Similarly, the second clade was separated two branches including a species $C$. almehensis samples of Biflori series, and another poorly supported clade (Bootstrap 53\%). In particular, two autumn flowering species C. speciosus isolate Deylaman and C. speciosus isolate Roudbar, belonging to Speciosi series, are in a very poorly supported clade (bootstrap value of 36\%) associated with $C$. sp. Sonqor and $C$. sp. Harsin, according to high bootstrap values (96\%).

Therefore, this last evidence could not be fully confirmed. Indeed, Petersen et al. (2008) stated that Reticulati and Biflori groups have revealed some of the most challenging taxonomic problems in this genus. Since Crocus sp. Sonqor and Crocus sp. Harsin samples are autumn flowering species which were collected in a geographical area close to Kermanshah and have a high bootstrap value (96\%), thus they are in the same branch.

The analysis indicated a close relationship between crop saffron and four wild species of Crocus caspius, Crocus michelsonii, $C$. sp. Eslamabad-e Gharb and C. cancellatus. According to this study among 13 available sample, the wild species $C$. cancellatus and $C$. sp. Eslamabad-e Gharb were considered as the closest species to saffron in Iran (C. sativus), based on genetic distance and dendrogram obtained by analyzing four barcode genes (trnH-psbA + rbcL + matK + ITS) separately and all together.

However, according to IRAP data (Alavi-Kia et al. 2008), this analysis revealed a close relationship between crop saffron and three wild species, C. almehensis, C.michelosnii, and $C$. cancellatus. Accordingly, this study considered C. almehensis and C. michelosnii as the closest relatives of modern saffron and possible ancestors of this species. It implies the involvement of a geographical relationship in the production of these three species. Finally, Nemati et al. (2018) analyzed sequences of two chloroplast (trnL-trnF, matK-trnK) and three nuclear (TOPO6, ribosomal DNA ETS and ITS) marker regions to infer phylogenetic relationships among series Crocus, making an autotriploid origin of C. sativus from C. cartwrightianus very likely.

\section{References}

Alavi-Kia SS, Mohammadi SA, Aharizad S and Moghaddam M 2008. Analysis of genetic diversity and phylogenetic relationships in Crocus genus of iran using inter- retrotransposon amplified polymorphism. Biotech \& Biotechnol Eq. 22: 795-800.

Asadi F, Dezhsetan S, Ghahramanzadeh R, Razmjou J and Alebrahim MT 2015. DNA barcoding of some medicinal plants. Crop Biotech. 10: 31-40

Casiraghi M, Labra M, Ferri E, Galimberti A and De-Mattia F 2010. DNA barcoding: A six-question tour to improve users' awareness about the method. Brief in Bioinform. 11: 440-453.

Dellaporta SL, Wood J and Hicks JB 1983. A plant DNA mini preparation: version II, Plant Mol. Biol. Rep. 1: 19-21.

Gismondi A, Fanali F, Labarga JMM, Caiola MG and Canini A 2013. Crocus sativus L. genomics and different DNA barcode applications. Plant Sys. Evol. 299: 1859-1863.

Gismondi A, Rolfo MF, Leonardi D, Rickards O and Canini A 2012. Identification of ancient Olea europaea L. and Cornus mas L. seeds by DNA barcode. C. R. Biologies. 335: 472-479. 
Hollingsworth ML, Clark AA, Forrest LL, Richardson J, Pennington RT, Long DG, Cowman R, chase M, Gaudeul M, Hollingsworth PM 2009. Selecting barcoding loci for plants: Evaluation of seven candidate loci with species level sampling in three divergent groups of land plants. Mol. Ecol. Resour. 9: 439-457.

Harpke D, Meng S, Rutten T, Helmut K, Frank B 2013. Phylogeny of Crocus (Iridaceae) based on one chloroplast and two nuclear loci: Ancient hybridization and chromosome number evolution. Mol. Phylogenet. Evol. 66: 617-627.

Hebert PDN, Ratnasingham S and Dewaard J R 2003. Barcoding animal life: cytochromec oxidase subunit 1 divergences among closely related species. Proc. R. Soc. Lond. B. Biol. Sci. 270: 96-99.

Huang WJ, Li FF, Liu YJ and Long CL 2016. Identification of Crocus sativus and its adulterants from Chinese markets by using DNA barcoding technique. Iran. J. Biotechnol. 13: 36-42.

Kim S, Misra A 2007. SNP genotyping: technologies and biomedical applications. Annu. Rev. Biomed. Eng. 9: 289-320.

Kim S-C, Chunghee L and, Mejias JA 2007. Phylogenetic analysis of chloroplast DNA matK gene and ITS of nrDNA sequences reveals polyphyly of the genus Sonchus and new relationships among the subtribe Sonchinae (Asteraceae: Cichorieae). Mol. Phylogenet. Evol. 44: 578-597.

Kress WJ, Erickson DL, Jones FA, Swenson NG, Perez R, Sanjur O and Bermingham E 2009. Plant DNA barcodes and a community phylogenyof a tropical forest dynamics plot in Panama. Proc. Nat. Acad. Sci. USA. 106: 18621-18626.

Kress WJ, Wurdack KJ, Zimmer EA, Weigt LA and Janzen DH 2005. Use of DNA barcodes to identify flowering plants. Proc. Nat. Acad. Sci. USA. 102: 8369-8374.

Larsen B, Orabi J, Pedersen C and Ørgaard M 2015. Large intraspecific genetic variation within the SaffronCrocus group (Crocus L., Series Crocus; Iridaceae). Plant Syst. Evol. 301: 425-437.

Mathew B 1982. The Crocuses: a revision of the genus Crocus (Iridaceous). B T Batsford, London

Nemati Z, Blattner FR, Kerndorff H, Erol O and Harpke D 2018. Phylogeny of the saffron-Crocus species group, Crocus series Crocus (Iridaceae). Mol. Phylogenet Evol. 127: 891-897.

Okuyama Y and Kato M 2009. Unveiling cryptic species diversity of flowering plants: successful biological species identification of Asian Mitella using nuclear ribosomal DNA sequences. BMC Evol Biol. 9: 105.

Petersen G, Seberg O, Thorsoe S, Jorgensen T and Mathew B 2008. Phylogeny of the genus Crocus (Iridaceae) based on sequence data from five plastid regions. Taxon 57: 487-499.

Rahimmalek M, Bahreininejad B, Khorami M and Sayed Tabatabaei BE 2009. Genetic diversity and geographical differentiation of Thymus daenensis, an endangerd medicinal plant, as revealed by Inter Simple Sequence Repeat (ISSR) markers. Biochem. Genet. 47: 831-842

Rohwer JG 2000. Toward a phylogenetic classification of the Lauraceae: Evidence from matK sequences. Syst. Bot. 25: 60-71.

Schwei T 2015. DNASTAR Lasergene 12.2 Software Achieves High Accuracy (Press release). DNASTAR.

Tamura K, Peterson D, Peterson N, Stecher G, Nei M and Kumar S 2011. MEGA5: Molecular evolutionary genetics analysis using maximum likelihood, evolutionary distance, and maximum parsimony methods. Mol. Biol. Evol. 28: 2731-2739.

Wolfe KH, Li WH and Sharp PM 1987. Rates of nucleotide substitution vary greatly among plant mitochondrial, chloroplast, and nuclear DNAs. Proc. Nat. Acad. Sci. USA 84: 9054-9058.

Zubor AA, Suranyi G, Gyori Z, Borbely G and Prokisch J 2004. Molecular biological approach of the systematics of Crocus sativus L. and its allies. Acta Hort 650: 85-93.

(Manuscript received on 28 November, 2018; revised on 27 July, 2019) 